\title{
The Caring Self: The Older Adult Care Providers' Experiences
}

\author{
Daisy Palompon \\ Cebu Normal University \\ daisypalompon@gmail.com
}

\begin{abstract}
This study explored the meaning of the caring experiences of nurses and caregivers in a private older adult facility. Using Hermeneutic phenomenology, the study interviewed ten informants, and the data gathered were analyzed using NVivo, a qualitative data analysis software. Findings revealed four themes: a) My mission, my passion; b) My Challenge, my victory; c) Caring beyond boundaries and d) Caring beyond self. The study concluded that the caring self of the care providers consists of three dimensions of caring: confident care, strategic care, and enduring care.
\end{abstract}

Keywords: caring self, older adults, care agent, care providers

\subsection{Introduction}

Older adults' population is projected to increase globally (Asian Development Bank, 2017). According to National Institutes of Health (2016), 8.5 percent of people worldwide (617 million) are aged 65 and over. In the Philippines, there is also a growing percentage of older adults. Senior citizens (aged 60 years old and over) made up 6.8 percent of the 92.1 million household population in 2010, higher than the 6.0 percent recorded in 2000. Among the senior citizens, females (55.8 percent) outnumbered the males (44.2 percent) (Philippine Statistics Authority, 2012).

The focus on the elderly population requires attention to their specialized care. The Philippines is known for its culture of caring for the elderly by the family. Even with this prevailing culture, there has been an increasing demand for elderly care facilities. The nurses and care providers in these facilities provide good source of information on the experiences of caring for older adults. The care provided for young and adult clients differs from the care provided to an older adult since the latter are often besieged with physical limitations and incidence of dementia. Despite their old age, they often exhibit their sense of authority and demand respect from care providers. Hence, in dealing with the gerontology care, care providers need more patience and understanding. The meaning of caring for older persons can explain what makes it different from other types of caring and provide a clear distinction of why nurses and caregivers opt to work in an elderly facility. Hence, care for older adults should not be stereotyped as the same as other clients since there is a need for a unique approach in their care. Knowing the elements of caring and how this transpires between the elderly client and the care provider whether he/she is a nurse or a caregiver would provide relevant information in describing a care providing 
framework for the older adult population. Integrated in this framework is the influence of culture on the way older adults are cared for in the Filipino setting. The care providers' cultural beliefs and practices in the care of older clients are also relevant concepts that need to be immersed in the study. It is the premise of this study that understanding the experiences of care providers of the elderly is essential in providing quality care to older adults who are the care recipients and utilizers of health care services. They have frontline experience in caring for older adults who rely upon them for multiple needs (Cohen, Colantonio, \& Vernich, 2002).

Every time a nurse -to-patient interaction is made, the concept of caring in the health profession takes place. "The nurse enters the world of the patient to know the patient as a caring person, and that it is from this 'epistemology' that the caring of nursing unfolds". Caring can change a patient's sense of well-being. "Caring may occur without curing, but curing cannot occur without caring" (Vance, 2003). In a caring universe, the significant event that needs to be captured is the ideas of what and how caring for older adults are best exemplified in the experiences of people who have immersed themselves in the caring phenomenon. Hence, this study considered the actual experiences, meaning of experiences, and insights caregivers have acquired throughout their years of older adult caring.

\section{Phenomenological Approach}

The methodological and theoretical perspectives of the study use the phenomenological lens of Martin Heidegger. Heidegger considered the ontological stance on what is nature of being which led to the idea, that ontology and phenomenology could not be separated from each other (Ong, Palompon, Amoin, \& Ygay, 2015). Tan, Wilson, and Olver (2009) concluded that phenomenology means "to let that which shows itself be seen from itself in the very way in which it shows itself from itself".The discussion on revealing and unveiling are descriptive, interpretive, and linguistic and therefore that of hermeneutics. A typical sense of a person is to create interpretations of what he or she sees or hears, but these interpretations are either changed or sustained when the interaction takes place. The encounter in the lived experience of the care providers of older persons provides the core of the study. Hence, the focus of this hermeneutic investigation is the care providers' experiences in caring for the elderly in a facility.

The care mentioned in this study is on older adults' care experiences. The care experiences of vulnerable and frail individuals, who had once contributed to society's welfare, became well respected in the community. The phenomenon of caring requires a relationship between the care providers and their elderly clients as well as their family on how they relate with each other and the people involved in the caring process. This study utilized narratives that illustrate the meaning and essence of experiences of care providers for older persons.

\subsection{Methodology}

This study explored the meaning of the caring experiences of nurses and caregivers working for the elderly in a privately-owned home for the aged run by a religious group which was established nineteen years ago. The meaning of caring serves as the core component and the essential aspect of elderly care. Gaining insights and meanings of elderly care in life is an intensive understanding of the phenomenon of caring. 
A most appropriate approach used in this aspect of the study is interpretative phenomenology or hermeneutics guided by the philosophy of Martin Heidegger. Interpretative phenomenology aims to uncover hidden meanings (Dowling, 2004). Heidegger's philosophy grounds on the study of authentic experiences of persons. "It is considered that people's realities are influenced by the world they live in as the focus of interpretive phenomenology is to explore the lived experiences". Hence, there is also a need for the researcher to consider the influence of social and political contexts (Tuohy, Cooney, Dowling, Murphy \& Sixmith, 2013). The establishment of hermeneutic circles in the study is most aspired as the interpretation through understanding is made through an iterative, continuous re-examination of the experiences that unravel their true meaning. This aspect of the study took place in the same older adult care facility.

According to Groenewald (2004), the phenomenon being studied dictates the method and even the type of participants, and not viceversa. The participants of the study are the nurses and caregivers who provided actual care of the elderly and should have done so for at least one year and have provided their written consent to be part of the study. Ten (10) participants were purposively selected as they qualified according to their years of employment in the facility and their willingness to volunteer as participants of the study. They were interviewed and the number of participants was dependent on the data saturation level. The good judgment of the researcher determines whether saturation has been reached or not. Every interview was audio- recorded, labeled, and transcribed before the analysis. The researcher also took field notes during the interviews. These notes presented the context at which the information was provided, which could not be captured in the transcriptions or audio recordings. They also served as a "step towards data analysis".

Data analysis utilized the interpretative phenomenological analysis (IPA). This approach assumes that the researcher is interested in learning the participants' world in the form of beliefs and constructs, which the participant presents in his or her story. Its central aim is to understand the content and complexity of the meaning of elderly caring by the care providers. IPA was done by engaging in an "interpretive relationship with the transcript". Steps in IPA were utilized starting with the identification of the theme in the first case, connecting the themes, continuing the analysis with other cases and the final step is the movement from the final themes to a write-up with the final statement outlining the meanings inherent in the participants' experiences (Smith \& Osborn, 2007).

In 1985, Lincoln and Guba's were used to establish the trustworthiness of the study. They identified four aspects to consider in establishing rigor, which is credibility, transferability, dependability, and conformability. The rigors of the research further utilized Rolfe's (2006) criteria such as credibility, dependability, conformability, transferability, and authenticity. Credibility was achieved through the recruitment and interview of participants who can well describe and have had direct experience in caring for the elderly. This was further established through the use of field notes which were used to capture contextual and transcriptions. The prolonged engagement and exposure of the researchers in the actual caring setting for elderly also provided depth in the data gathered. Dependability was attained by performing audit trail, providing detailed 
description of the research methods undertaken, and peer and expert evaluation of the procedures and results. Conformability satisfied by the researcher's reflexivity, that is, "the process in which researchers are conscious of and reflective about the ways in which their questions, methods and very own subject position might impact on the data or the psychological knowledge produced in a research study". Transferability or the "fittingness" was established by providing rich and in-depth description of the setting, of the informants and of the experience of the informants as well as strict compliance to the inclusion criteria. The careful abstractions of the concepts observed with the constant comparison of reports of the informants and the concepts generated made the authentic descriptions easily relatable and comprehensible. Authenticity was observed with the researcher presenting all values, views and differences. Although hermeneutics allowed subjectivity of the researcher to come into the analysis and interpretation of the data, the researcher practiced holding off of judgment and biases. Reflexive notes or written observations of gestures, actuations or facial expressions of the participants during the interview with the personal reflections of the researcher and the research assistants also helped in the development of insights of the researcher.

The conduct of the study observed strict ethical considerations. An accredited Ethics Review Board approved the protocol of the study. The study's purpose and design were presented to the management and staff before the start of the study, and a sample handout with brief informative text describing the study's aim, methodology and a copy of the informed consent was provided. Administrative consent and individual participants' informed consent were provided to the researcher before the start of the study.

Data were analyzed using NVIVO software. Data were inductively coded by creating nodes relevant to the objective of the study and relevant texts were dragged to the corresponding nodes. Data were then clustered using word cloud and word tree to determine the occurrence of terms which are synonymous to each other. From the clusters, themes and sub-themes were developed.

\subsection{Results and Discussion}

During the data collection period, the informants shared their experiences, views, feelings, and emotions when asked about their caring experiences with the elderly staying in the facility. From the recorded interviews, the researchers identified different themes from the outcomes of concept synthesis and analysis. The perspectives of the caring experiences of the informants as interpreted by the researcher served as basis in the generation of the themes.

\section{Theme 1. My Mission, My Passion (Confident Care)}

This theme is generated from two sub-themes: a) Knowing care, talking care; and, b) you and your family: my world of caring. These two sub-themes provide the structural scaffolds in arriving at the theme.

The care informants believed that the purpose at which they were placed in the elderly facility is a mission that they have to accomplish. It is not just working but a calling beyond the job as they believed that despite their previous employment and the difficulties they encountered in caring for the older adults, they still pursued working in the facility. Some of the nuns who are the nurses in the facility consider their vocation for the sick elderly 
as their mission in life. The non-religious care providers also expressed that they consider their work in the elderly facility as their life's mission.

The caring experiences taught to develop the sense of accomplishing something good for the elderly and develop the passion of caring for these special people. The many years of caring for the elderly have provided them a sense of direction on what to do with their career life. They have integrated into their job as care provider the love for work and commitment to provide better care for the older persons.

\section{Knowing Care, Practicing Care}

In their daily care activities, the informants expressed the importance of one's knowledge in caring which enables them to easily express and share their caring experiences. The competencies acquired by the care providers of older adults enabled them to manifest these characteristics in their manner of giving care to older clients. Caring for the older persons requires training and learning the routine care activities. They also believed that knowing care is a dose of confidence through personal faith and practices.

The ownership in doing the care for the elderly is an indicator of the care providers' immersion and mastery of the routine activities of care (Fagerberg \& Kihlgren, 2001). There is a need for the care providers to demonstrate competence in caring to establish trust and confidence of the older adults to the care provider. The clients need to appreciate the confidence and competence of the care provided to them so that they will feel that they are well taken care of.

\section{You and Your Family: My World of Caring}

The informants consistently mentioned the role of the family in their care for the older adult. It is part of the caring routine of the informants to invoke the family as part of their daily work provision. The elderly's family takes the role of an invisible inspiration, which helps the care providers in the provision of care. The informants appreciate the magic that the names of the family members evoke from the elderly and the happiness they feel when the informants ask to relate stories of their lives and their family members. The engagement of family implies that as care providers, they should not only learn the technical aspects of their job but they should also know their patients on a personal level, establishing rapport and knowing about the history of the patients. The experiences encourage the nurse and caregivers to provide care to older persons according to their unique individual needs.

\section{Theme 2. My Challenge, My Victory (Enduring Care)}

This theme encompasses the experiences of the informants in caring for the older adults characterized with difficulties interspersed with successes. The sub-themes: a) A Baptism of fire, and b) Little triumphs, bigger rewards exemplify enduring care. The problems of the care for elderly can be attributed to the physical limitations due to aging, at the same time, the reduction of mental or cognitive functioning which is in most cases accentuated in the elderly with Alzheimer's disease (Young, 2003).

\section{Difficulties of Caring for Older Clients}

Working in an elderly facility has often been described by the informants as more difficult than caring for children. When they started working in the facility as elderly care providers, they have gone through a lot of adjustments, including physical and emotional pains. The sharing of the 
participants during the interview depicts the experiences they went through. The life of the care providers is tested by the older adults' mood or emotional state. It is indeed a trying experience for them. Hence, only a few remained in the job. These mood changes might be due to their mental decline or physical discomforts, which make them irritable. However, the constant understanding of the care providers for the patient enables them to cope with the difficulties they have encountered.

\section{The Joys and Satisfaction of Caring}

The lives of the care provider with the elderly are not only characterized by difficulties. They also expressed instances of good memories and satisfaction afforded to them by their elderly clients. There were moments where the hardships were rewarded with smiles, and thank yous. They consider these instances as little successes, which made them appreciate their job as elderly care providers. The happiness that the care providers felt as they took care of the older persons is for them an affirmation of the job they have done to the clients. The little improvements in the attitude of the clients gave them the satisfaction that every care provider expects to happen in his or her caring experience. These are little perks and rewards in the lives of the care providers which makes their job more satisfying. When clients appreciate their care, this provided an affirmation that what they are doing is right and creates a sense of confidence and empowerment on them.

\section{Theme 3. Caring Beyond Boundaries (Strategic Care)}

In the years of working with the older adults, the care providers were able to develop the care strategies that helped them in dealing with clients. These are personal strategies which they learned as they worked in the facility. These are indications of the advanced caring competencies where they used life's lessons as their guide in their care practices. These are the care providers' strong commitment to their jobs and the care strategies utilized. This theme is substantiated with the two sub-themes: a) Tales of Times, and b) Being There.

\section{Tales of Times}

The most common strategy of care shared by the informants is the use of storytelling based on the historical life of the elderly client or even the life story of the care providers themselves. This practice is affirmed by a study finding that life stories helped practitioners to view patients as human beings, to understand them more fully and develop close relationships with the patients' families (Clarke, Hanson, \& Ross, 2003). The use of this strategy also entertained the care providers, which made the care relationship more personalized and person-centered. The use of storytelling is also supported by another strategy that is commonly described as flattery (ulo-ulohan). This strategy is the use of words for the elderly to calm down. This strategy is a way to appease the elderly and to divert the attention of the elderly and forget their anger or resentment towards doing something. Avoiding confrontation and giving her time while maintaining close supervision when the elderly is angry is also another strategy used and showing one's affection towards the elderly often give them a feeling of affection and acceptance.

\section{Being There}

Loneliness is a feeling among older persons admitted in facilities. They long for their family members and people whom they are fond of. The only people who can address that need are the 
care providers. Despite the busy schedule, the care providers still find time to be with their clients. Being there means physically being with the client with their hearts and minds engaged in the care of the elderly.

\section{Theme 4. Caring Beyond Self (Caring Self)}

The care providers shared their experiences on elderly caring as their life. It is "their life" because they considered these as their care for their old parents or even their aspiration of how they will be cared for when they also become old without expecting in return. This theme is generated through the three sub-themes: a) Lola, Mother, and Self: 3D Mirroring; b) Time, Patience and Love for Lola: Rolled into One; and c) My Faith, My Commitment.

\section{The Inspirations of Caring}

The experiences of caring for older persons mirror the three (3) reasons for caring: the actual care for the elderly client, the care for ones' elderly parents; and the personal aspiration of the quality of care that can be provided to them when they grow old. It is care dedicated towards somebody whom they are close with, a making up for the loss and for doing what has not been done before. This experience strengthens their commitment to their work and encourages them to be more affectionate care providers. There is a personal quality in the care provided because of the mirroring that the care providers experienced. The care providers also likened their job to taking care of their family members. Treating the elderly as one's family members enables them to accept each client as he or she is.

\section{Personal Investment in Caring}

According to the care providers, to give quality care to the elderly, the significant elements to consider are time for the elderly, patience in times of difficulties and love when the elderly becomes uncontrollable. This is one perspective which care providers have not realized as demands for the care of older adults. The care providers need to stretch their patience when dealing with demented clients whose sense of authority still prevails. The sense of loss of control which demented clients experienced with the desire to preserve their selfesteem is a situation that requires much control of one's temper, tolerance and an understanding of the clients. Elderly care requires time to be with the client to focus on the human perspectives of care rather than focusing on the technical aspects of caring. Patience requires time, and patience needs love. Care providers are provided with minimal compensation for their job, but despite these limitations, they prefer to stay and opt to continue caring for the older clients. These combinations of care make the self a caring person.

\section{My Faith, My Commitment}

The informants anchored their commitment to their work with their faith in God. They considered the service provided to the elderly as their share in the sacrifice of Jesus. Hence, their caring emanates from one's values placed on the life of a person. Their faith creates a foundation for the passionate caring they have demonstrated to their clients. The work is considered more than work itself but a call for service and sacrifice. They shared that the reason for staying as care provider is not due to financial reasons but more of spiritual. 
The experiences of the care providers for older adults were characterized by the themes generated such as my 1) mission, my passion; 2) my challenge, my victory; 3) caring beyond boundaries; and 4) caring beyond self. The first theme depicted the caregivers' experiences on their extent of competence in caring and the development of caring strategies brought about by the many years of caring experiences. This is a demonstration of how care providers gained their confidence in providing care. The challenges and the rewards that went with the caring experiences of the care providers developed in them a sense of enduring care which strengthens their commitment to their jobs. With the sense they have developed over the years, they were able to discover strategies of caring which are considered more of addressing personal rather than technical needs of older clients. These created the strategic care in them which developed in them a certain level of expertise of caring. And the value placed by the care providers to their job developed a sense of caring beyond them. The themes showed how the quality of a nurse or care provider affects the quality of care acquired by the clients. In the care of older adults, nurses and care providers are expected to be more aware, reflective and discerning of their experiences since caring for this type of clients require a more customized and grounded type of care. In the study of Schulz and Eden (2016), care giving experiences in older persons enable them to find meaning in their tasks, made them feel more confident and bring them closer to their care recipient. Although, it was emphasized that care giving experience is highly individual and dependent on the circumstances surrounding the care giving experiences. In another study in Nigeria, which focused on the experiences of caregivers of older persons, one of the common experiences is the caregivers' commitment to preservation of life (Faronbi, J., Faronbi, G., Adenika \& Ayobola, 2019). The pervading experience shows the sense of mission and commitment in the care for the older persons.

Based on the experiences of caring and the themes generated, the caring experiences focused on the caregiver himself or herself. The caring experiences of care providers among older adults influenced the persistence and patience in caring, as well as, the techniques and strategies developed by care providers. The care providers' experiences in older adult care ultimately focused on the self as the instrument delivering the quality of care for the clients. The uniqueness of the experiences of the care providers is a combination of certain caring factors, which emerged from the data. The aspects of confident, enduring and strategic care all focused towards the caring self of care providers. The core element of elderly care is the caring self which facilitates the provision of elderly quality of life or care satisfaction.

\subsection{Conclusion}

A substantive Theory on the Caring Self on Elderly Care provides that caring experiences of care providers among elderly generated themes which depict the competence of the care providers and the confidence they felt in caring for the elderly. The fulfillment of providing care lies on the sense of the self of the care provider. It is therefore recommended that in dealing with care providers in an elderly facility, the caring experiences and management be focused not only on the client but also on the care providers themselves as the success of the caring process lies on them. In as much as the development of confident care 
involves the knowledge and expertise of caring for older adults, it is recommended that appropriate professional preparation and continuing education be provided.

\section{References}

Asian Development Bank. (2017). Population and aging in Asia. Retrieved from http/www.adb. org/features/asia-s-growing-elderly.

Clarke, A., Hanson, E. J., \& Ross, H. (2003). Seeing the person behind the patient: Enhancing the care of older people using a biographical approach. Journal of Clinical Nursing, 12(5), 697-706.

Cohen, C. A., Colantonio, A., \& Vernich, Lee. (2002). Positive aspects of caregiving: Rounding out the caregiver experience. International Journal of Geriatric Psychiatry, 17, 184-188. doi: 10.1002/gps.561

Dowling, M. (2004). Hermeneutics: An exploration. Nurse Researcher, 11(4), 30-39.

Fagerberg, I., \& Kihlgren, M. (2001). Registered nurses' experiences of caring for the elderly in different health-care areas. International Journal of Nursing Practice, 7(4.), 229-236.

Faronbi, J., Faronbi, G., Adenika, J., \& Olaogun, A. (2019). Caring for the seniors with chronic illness: The lived experience of caregivers of older adults. Archives of Gerontology and Geriatrics, (82.), 8-14.

Groenewald, T. (2004). A phenomenological research design illustrated. International
Journal of Qualitative Methods, 3(1). Article 4. Retrieved from http://www.ualberta.ca/ iiqm/ backissues/3_1/pdf/groenewald.pdf

Lincoln, Y. S., \& Guba, E. G. (1985). Naturalistic inquiry. Newbury Park, CA: SAGE.

National Institutes of Health. (2016). World's older population grows dramatically. Retrieved from https://www.nih.gov/news-events/newsreleases/worlds-older-population-growsdramatically.

Ong, M. L., Palompon, D. R., Amoin, C., \& Ygay, G. (2015). Terrors, losses and new lives of disaster survivors: A phenomenological inquiry. Asian Journal of Health, 5(1), 28-47

Philippine Statistics Authority [PSA]. (2012). The 2010 census of population and housing reveals the Philippine population at 92.34 million. Retrieved from https://psa.gov. ph/content/2010-census-population-andhousing-reveals-philippine-population-9234million.

Rolfe, G. (2006). Validity, trustworthiness and rigour: quality and the idea of qualitative. Methodological Issues in Nursing Research, 53.(3.), 304-310. Blackwell Publishing. Retrieved from http:// garyrolfe.net/documents/validitytrustworthiness. pdf

Shulz,R. \& Eden, J. (2016). Families caring for an aging America. National Academies Press, 2-6.

Smith, J. A., \& Osborn, M. (2007). Interpretative phenomenological analysis. In J. A. Smith 
(Ed.), Qualitative psychology: A practical guide to research methods (2nd ed.) (pp. 53-80). Thousand Oaks, CA: SAGE.

Tan, H., Wilson, A., Olver, I. (2009). Ricoeur's theory of interpretation: An instrument for data interpretation in hermeneutic phenomenology. International Journal of Qualitative Methods, 8(4), 1-15. Retrieved from https://ejournals.library.ualberta.ca/index. php/IJQM/article/view/4049/5889.

Tuohy, D., Cooney, A., Dowling, M., Murphy, K., \& Sixmith, J. (2013). An overview of interpretive phenomenology as a research methodology. Nurse Researcher, 20(6), 17-20.

Vance, T. (2003). Caring and the professional practice of nursing. Journal of Nursing. Retrieved from https://www.scribd.com/doc/102058375/ Caring-and-the-Professional-Practice-ofNursing-docx.

Young, H. M. (2003). Challenges and solutions for care of frail older adults. Online Journal of Issues in Nursing, 8(2), Manuscript 4. Retrieved from www.nursingworld.org/MainMenuCategories/ ANAMarketplace/ANAPeriodicals/OJIN/ TableofContents/Volume82003/No2May2003/ OlderAdultsCareSolutions.aspx. 